\title{
Exotic Option Prices Simulated by Monte Carlo Method on Market Driven by Diffusion with Poisson Jumps and Stochastic Volatility
}

\author{
Magdalena Broszkiewicz and Aleksander Janicki \\ Mathematical Institute, University of Wrocław, \\ pl. Grunwaldzki 2-4, 50-384 Wrocław, Poland \\ \{janicki, msoboc\}@math.uni.wroc.pl \\ http://www.math.uni.wroc.pl/ janicki
}

\begin{abstract}
We consider a broad class of stochastic models of a financial market generalizing the classical Black-Scholes model, which comprise both: stochastic volatility of Brownian type and jumps at random times. We restrict ourselves to the model, where volatility is described by the diffusion which comprises the Heston stochastic volatility defined as a diffusion of Brownian type and the Poisson jump diffusion. We provide an argument that such models perfectly match typical real-life financial phenomena comparing the so-called logarithmic returns.

Applying computer simulations methods we investigate the dependence of prices of a few selected contingent claims (specifying some different options) on the parameters of our stochastic model.
\end{abstract}

\section{The Market Model}

Consider a financial market where two assets $S_{0}$ and $S$ are traded up to a fixed horizon $T$.

Let $(\Omega, \mathcal{F}, P)$ be a probability space and let $\{\mathcal{F}(t)\}_{0 \leq t \leq T}$ be a P-augmented right-continuous filtration.

The riskless asset price is given by

$$
S_{0}(t)=\exp (r t), \quad \forall t \in[0, T],
$$

where $r$ is non-negative deterministic risk free interest rate. The dynamics of risky asset's price is given by

$$
\begin{aligned}
d S(t) & =S\left(t_{-}\right)\left\{\mu d t+\sigma(V(t))\left[\sqrt{1-\rho^{2}} d W(t)+\rho d W^{\sigma}(t)\right]\right. \\
& \left.+\alpha_{1} d \widetilde{N}^{\lambda_{1}}(t)+\alpha_{2} d \widetilde{N}^{\lambda_{2}}(t)\right\} \\
d V(t) & =\eta d t+\gamma d W^{\sigma}(t)
\end{aligned}
$$

where $\left(W, W^{\sigma}\right)^{\prime}$ is two dimensional Brownian motion, $\widetilde{N}^{\lambda_{i}}$ are independent compensated Poisson process (ie. $\left.\tilde{N}^{\lambda_{i}}(t)=N^{\lambda_{i}}(t)-\lambda_{i} t\right),\{V(t)\}_{0 \leq t \leq T}$ is the 
volatility process, $\rho$ is the correlation between asset process and its volatility, $\rho \in[-1,1], \mu, \eta, \gamma$ are constants, $\sigma$ is a function that satisfies

$$
\exists \underline{\sigma}, \bar{\sigma}>0 \quad \forall z \in \mathbb{R} \quad \underline{\sigma} \leq \sigma(z) \leq \bar{\sigma} .
$$

We take two independent Poisson processes to assure that both jumps up and down would be available. To achieve that it is necessary to choose parameters $\left|\alpha_{1}\right|<1$ and $\left|\alpha_{2}\right|<1$ of the different signs. To explain this model we suggest

(a)

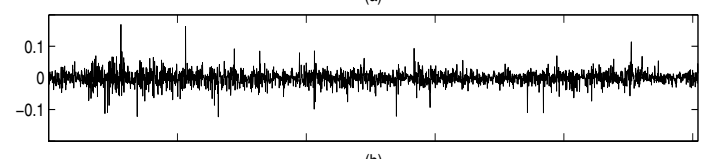

(b)

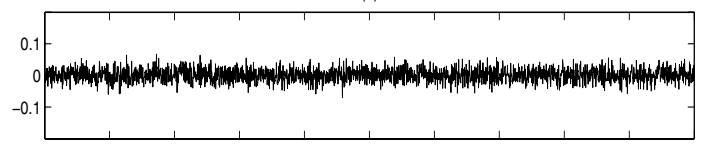

(c)

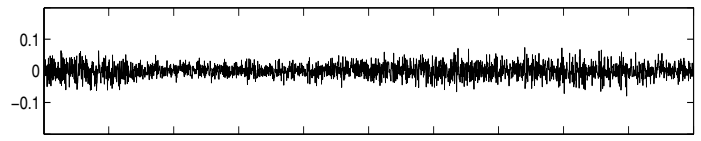

(d)

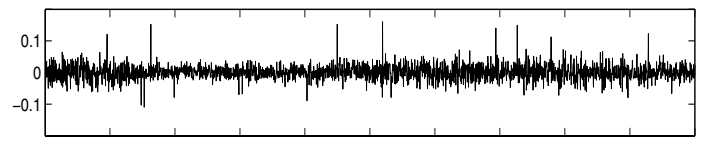

Fig. 1. Asset's price returns for various market model. On picture (a) there are real logarithmic returns of prices of IBM's stocks. Remaining pictures show logarithmic returns of prices modelled in various way: (b) simple diffusion (like Black-Scholes model), (c) stochastic volatility (like Heston model), (d) stochastic volatility and Poisson jumps

to compare the examples obtained at Fig.1. Looking at (b) and (c) we see that stochastic volatility improves the model and makes it more accurate but only (d) reflects all phenomenon which are characteristic to prices behavior. It is well known that absence of arbitrage which is equivalent to existence of martingale measure, is absolutely necessary to compute the fair price of any option. In this new measure the process of discounted price of risky asset should be a martingale. On the market defined above the Radon-Nikodym's derivative can be defined as follow

$$
\begin{aligned}
Z(t) & :=\left.\frac{d \mathbb{Q}}{d \mathbb{P}}\right|_{\mathcal{F}(t)}=\exp \left\{-\int_{0}^{t} \theta(s) d W(s)-\frac{1}{2} \int_{0}^{t} \theta(s)^{2} d s\right\} \\
& \times \exp \left\{-\int_{0}^{t} \nu(s) d W(s)-\frac{1}{2} \int_{0}^{t} \nu(s)^{2} d s\right\} \\
& \times \exp \left\{\ln \left(1-\phi_{i}\right) N^{\lambda_{i}}(t)+\lambda_{i} \phi_{i}\right\}
\end{aligned}
$$


where $\theta$ and $\nu$ are adapted to $\{\mathcal{F}\}$ and satisfy the integrability conditions $\int_{0}^{T}(\theta(t))^{2} d t<\infty$ and $\int_{0}^{T}(\nu(t))^{2} d t<\infty$. From the condition $\mathbb{E}(Z(T))=1$ we obtain

$$
\left(\theta(t) \sqrt{1-\rho^{2}}+\rho \nu(t)\right) \sigma(t)+\lambda_{1} \phi_{1} \alpha_{1}+\lambda_{2} \phi_{2} \alpha_{2}=\mu-r \quad 0 \leq t \leq T \quad \text { a.s. }
$$

The processes $\theta$ and $\nu$ are interpreted as the risk premiums connected with the Brownian motions $W$ and $W^{\sigma}$ respectively. The premium for the risk related to Poisson processes $N_{i}^{\lambda}$ are $\phi_{i}$.

Having only one such equation we are not able to find the new measure uniquely. After Merton we can assume that the premiums coming from the Poisson processes disappear. But even than we can write the process $\theta$ only dependent on process $\nu$.

According to Girsanov's theorem we obtain $W_{0}(t)$ and $W_{0}^{\sigma}(t)$ as new Wienner processes and $\widetilde{N}^{L_{i}}(t)$ as a new compensated Poisson processes (with intensity $L_{i}$ ). Since only one risky asset is traded, the premiums are not unique and nor is the martingale measure.

Applying new processes to the discounted asset price we obtain

$$
\begin{aligned}
d\left(\frac{S(t)}{S_{0}(t)}\right) & =\frac{S\left(t_{-}\right)}{S_{0}(t)}\left\{\sigma(V(t))\left[\sqrt{1-\rho^{2}} d W_{0}(t)+\rho d W_{0}^{\sigma}(t)\right]\right. \\
& \left.+\alpha_{1} d \widetilde{N}^{L_{1}}(t) \alpha_{2} d \widetilde{N}^{L_{2}}(t)\right\}
\end{aligned}
$$

It is easy to see that the above process is a supermaringale and since $\sigma$ satisfies the (3) condition it is also a martingale.

\section{Option Prices}

It is well known that the option price with payout function $\psi(S(T))$ is ruled by the equation

$$
E C C(t)=S_{0}(t) \mathbb{E}^{\mathbb{Q}}\left[\frac{1}{S_{0}(T)} \psi(S(T)) \mid \mathcal{F}(t)\right]=\frac{1}{H(T)} \mathbb{E}^{\mathbb{P}}[H(T) \psi(S(T)) \mid \mathcal{F}(t)]
$$

where $H(t)=\frac{Z(t)}{S_{0}(t)}$ is a state density price.

Now substituting $S_{0}(t)$ according to (2), and knowing that $H(0)=0$ we obtain the option premium with payout function $\psi(S(T))$.

$$
E C C(0)=e^{-r T} \mathbb{E}[Z(T) \psi(S(T))]
$$

Having the solution in the form of expectation like in (6) , the easiest way to obtain results is using the Monte Carlo method.

In all examples containing stochastic volatility we assume that the function $\sigma$ has the form $\sigma(z)=z, \quad \underline{\sigma} \leq z \leq \bar{\sigma}$, And $\sigma$ have the values $\underline{\sigma}, \bar{\sigma}$, respectively outside the above interval. 
From the stochastic exponent theorem of we can compute the price of the stock in the moment of maturity $(t=T)$.

$$
\begin{aligned}
S(T) & =S(0) \exp \left\{\mu T-\frac{1}{2} \int_{0}^{T}(\sigma(V(s)))^{2} d s+\sqrt{1-\rho^{2}} \int_{0}^{T} \sigma(V(s)) d W(s)\right\} \\
& \times \exp \left\{\rho \int_{0}^{T} \sigma(V(s)) d W^{\sigma}(s)\right\} \exp \left\{\ln \left(1+\alpha_{1}\right) N^{\lambda_{1}}+\ln \left(1+\alpha_{2}\right) N^{\lambda_{2}}\right\} .
\end{aligned}
$$

Some results of simulations are presented in the table below.

Table 1. Prices of options simulated for various models of market: BS - Black-Scholes diffusion market, SV - stochastic volatility market, SV+P - stochastic volatility with Poisson jumps market

\begin{tabular}{|c|c|c|c|}
\hline Payout function & BS & SV & SV+P \\
\hline$(S(T)-K)^{+}$ & 17.8462 & 17.7726 & 17.5570 \\
\hline$X \mathbb{I}_{\{S(T)>K\}}$ & 10.7013 & 9.6102 & 10.3223 \\
\hline$S(T)-\min _{t}(S(t))$ & 28.5074 & 22.9462 & 28.7744 \\
\hline$\left(q^{\alpha}(S)-K\right)^{+}$ & 0.8136 & 3.1839 & 0.2556 \\
\hline$\left(K-M\left(T, T_{0}, S\right)\right)^{+}$ & 0.6422 & 1.1330 & 0.9097 \\
\hline
\end{tabular}

In all simulations the models are parameterized in the same way. First case is standard European option and parameters are chosen so to obtain similar premium. We assume exercise price $K=S(0)=100 \$$. Second line is binary option, which guarantee to pay amount $X=50 \$$ if the price of asset in the maturity time will be greater than $K=130 \$$. Third case is an lookback option (option depend on all trajectory, not only last moment). It pays the difference between price of stock at maturity time and minimal price of asset on all time horizon. Forth case is so-called quantile option $\left(q_{\alpha}(\cdot)\right.$ is a quantile of range $\left.\alpha\right)$ and the last is Asian option where $M\left(T_{0}, T, S\right)$ is the mean price on time horizon $\left(T_{0}, T\right), T_{0}>t_{0}=0$.

\section{References}

1. Bellamy N., Jeanblanc M.: Incompletness of markets diven by a mixed diffusion. Finance \& Stochastics 4 (2000) 209-222

2. León J. A., Solé J. L., Utzet F., Vives J. On Lévy processes, Malliavin calculus and market models with jumps. Finance \& Stochastics 6 (2002) 197-225

3. Protter, P.: Stochastic Integration and Differential Equations - A New Approach. Springer-Verlag, New York (1990)

4. Touzi N.: American Options Exercise Boundary When the Volatility Changes Randomly. Applied Matematics \& Optimization 39 (1999) 411-422 